\title{
Evidence for auxiliary anaerobic metabolism in obligately aerobic Zetaproteobacteria
}

\author{
Abhiney Jain ${ }^{1} \cdot$ Jeffrey A. Gralnick $\mathbb{D}^{1}$
}

Received: 2 September 2019 / Revised: 6 January 2020 / Accepted: 13 January 2020 / Published online: 22 January 2020

(c) Springer Nature Limited 2020

\begin{abstract}
Zetaproteobacteria are obligate chemolithoautotrophs that oxidize Fe(II) as an electron and energy source, and play significant roles in nutrient cycling and primary production in the marine biosphere. Zetaproteobacteria thrive under microoxic conditions near oxic-anoxic interfaces, where they catalyze Fe(II) oxidation faster than the abiotic reaction with oxygen. Neutrophilic Fe(II) oxidizing bacteria produce copious amounts of insoluble iron oxyhydroxides as a by-product of their metabolism. Oxygen consumption by aerobic respiration and formation of iron oxyhydroxides at oxic-anoxic interfaces can result in periods of oxygen limitation for bacterial cells. Under laboratory conditions, all Zetaproteobacteria isolates have been shown to strictly require oxygen as an electron acceptor for growth, and anaerobic metabolism has not been observed. However, genomic analyses indicate a range of potential anaerobic pathways present in Zetaproteobacteria. Heterologous expression of proteins from Mariprofundus ferrooxydans PV-1, including pyruvate formate lyase and acetate kinase, further support a capacity for anaerobic metabolism. Here we define auxiliary anaerobic metabolism as a mechanism to provide maintenance energy to cells and suggest that it provides a survival advantage to Zetaproteobacteria in environments with fluctuating oxygen availability.
\end{abstract}

\section{Introduction}

$\mathrm{Fe}(\mathrm{II})$ is an important electron and energy source in deep ocean biosphere. Zetaproteobacteria are the major Fe(II) oxidizing bacteria $(\mathrm{FeOB})$ in marine environments and shown to be widely distributed in deep ocean microbial communities [1-5]. Fe(III) oxyhydroxides produced as a by-product from neutrophilic $\mathrm{FeOB}$ may bind phosphate and other metals, thus affecting the biogeochemical cycling of multiple nutrients in addition to iron and carbon [6]. Therefore, understanding the metabolic strategies used by Zetaproteobacteria will help us understand key sources and flux of metabolic energy in the marine biosphere.

Supplementary information The online version of this article (https:// doi.org/10.1038/s41396-020-0586-6) contains supplementary material, which is available to authorized users.

Jeffrey A. Gralnick

gralnick@umn.edu

1 Department of Plant and Microbial Biology, BioTechnology Institute, University of Minnesota-Twin Cities, St. Paul, MN 55108, USA
Zetaproteobacteria are obligate chemolithoautotrophic $\mathrm{FeOB}$, relying on electrons generated from $\mathrm{Fe}(\mathrm{II})$ oxidation to generate reducing equivalents and ATP for carbon dioxide fixation. Mariprofundus ferrooxydans PV-1, the first cultivated member of the Zetaproteobacteria, was isolated from the Loihi seamount near Hawaii [1]. Very little is known about the metabolic strategies used by Zetaprotebacteria to survive in the environment, owing to the difficulty of laboratory cultivation and the lack of genetic tools. Cultivated isolates of Zetaproteobacteria have been shown to be strictly microaerophilic [1, 7-9]. Coupling oxygen reduction to $\mathrm{Fe}(\mathrm{II})$ oxidation is a challenge for $\mathrm{FeOB}$ because oxygen rapidly oxidizes $\mathrm{Fe}(\mathrm{II})$ abiotically at atmospheric concentrations. At micromolar and submicromolar oxygen concentrations the kinetics of abiotic $\mathrm{Fe}(\mathrm{II})$ oxidation by oxygen is slow enough to allow $\mathrm{FeOB}$ growth [10], and thus, Zetaproteobacteria are thought to thrive at oxic-anoxic interfaces where the oxygen concentrations are low $[1,5,7,9,10]$.

The depletion of oxygen by biotic and abiotic reactions at the oxic-anoxic interfaces where the oxygen concentrations are already very low can limit the continuous availability of oxygen. Under oxygen limiting conditions, anaerobic metabolism may provide a survival advantage to 
Zetaproteobacteria by providing maintenance energy in an oxygen independent manner. Because growth linked to anaerobic metabolism has not been shown in Zetaproteobacteria, we refer to this as "auxiliary anaerobic metabolism" where energy is generated for persistence and/or maintenance rather than growth. We analyzed the genomes of nine different Zetaproteobacteria isolates to search for genes known to be associated with anaerobic metabolism in central carbon metabolism, anaerobic respiration, and fermentation. We also validated activity of several genes via heterologous expression by complementing mutants in Shewanella oneidensis. Methods used for analyzing zetaproteobacterial genomes, S. oneidensis strain construction, and growth curves are described in the Supplementary information.

\section{Results and discussion}

\section{Zetaproteobacteria genomes contain genes for anaerobic metabolism}

All Zetaproteobacteria genomes analyzed contain multiple genes encoding for proteins known to be involved in anaerobic metabolism in other bacteria (Table 1). Pyruvate formate lyase (Pfl) and pyruvate ferredoxin oxidoreductase (Pfor) decarboxylate pyruvate to acetyl CoA under anoxic conditions to link glycolysis with the Krebs cycle, whereas pyruvate dehydrogenase is known to perform the same function under oxic conditions. The presence of $\mathrm{Pfl}$ encoding genes is strongly suggestive of anaerobic metabolism. Pfl from Escherichia coli has been reported to be extremely oxygen sensitive where exposure to oxygen leads to cleavage of the enzyme [11]. Pfl has been found to be essential for different anaerobic metabolisms in diverse bacteria [12-14]. Phosphotransacetylase (Pta) and acetate kinase (AckA) convert acetyl CoA to acetate and produce ATP by substrate level phosphorylation. Zetaproteobacteria are unable to grow heterotrophically on sugars and therefore are unlikely to use Pta and AckA for acetogenesis under oxic conditions as an overflow metabolism which occurs when sugars are metabolized at high growth rate in E. coli [15]. Moreover, the inability of Zetaproteobacteria to grow on acetate ([1], Table S2) limits the possible use of Pta and AckA for acetate assimilation under oxic conditions, but is consistent for a role of these proteins in auxiliary anaerobic metabolism. The Rnf complex, an anaerobic ferredoxindependent respiratory enzyme reported to be involved in ATP production and nitrogen fixation [16, 17], was also found to be encoded in all Zetaproteobacteria genomes analyzed. Under anaerobic conditions, the Rnf complex catalyzes the oxidation of reduced ferredoxin coupled to reduction of $\mathrm{NAD}^{+}$to form NADH and generation of

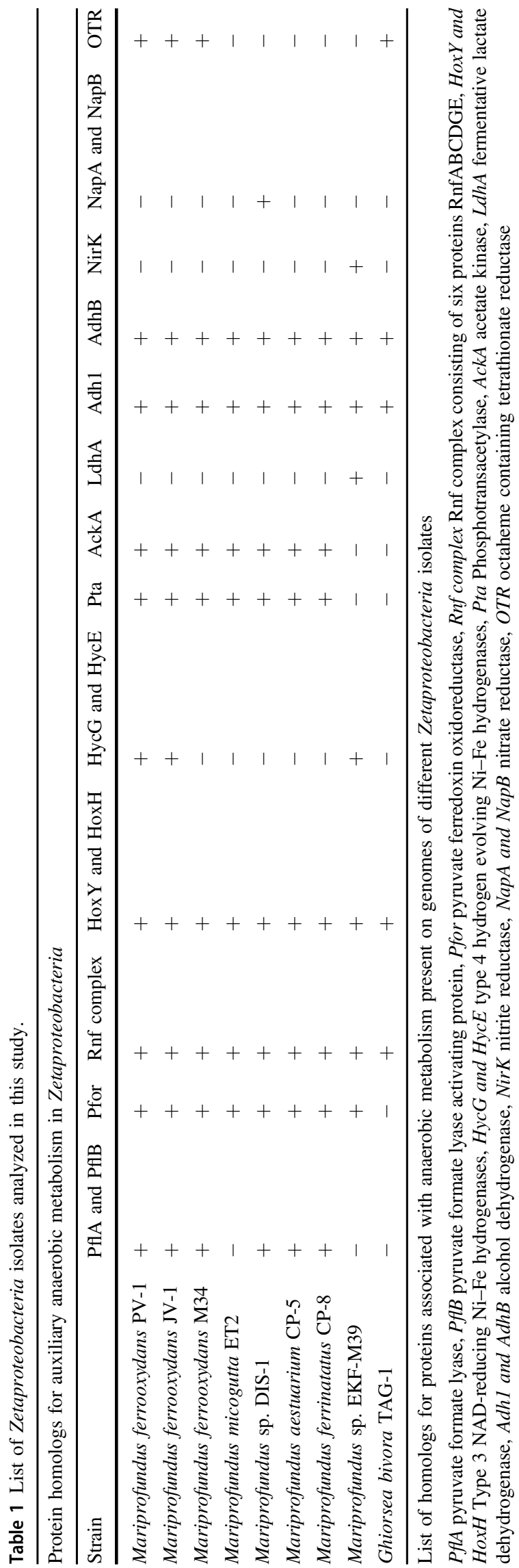


proton motive force (PMF). Fe(II) dependent oxygen reduction has been postulated as the only mechanism to produce PMF and ATP in Zetaproteobacteria [18]. Identification of genes encoding AckA and Rnf complex proteins suggest the presence of additional ATP and PMF generation mechanisms in Zetaproteobacteria.

Zetaproteobacteria have not been shown to grow heterotrophically, which limits the possibility of using exogenous organic carbon sources as substrate for anaerobic metabolism. One potential substrate for auxiliary anaerobic metabolism could be endogenous storage compounds. Zetaproteobacteria genomes were found to encode genes for synthesis and hydrolysis of glycogen (Table S1), which can serve as carbon and electron source under anoxic conditions. Anaerobic metabolism solely reliant on hydrolysis of endogenous storage compounds coupled to homolactic, heterolactic, and mixed acid fermentations has been described in other lithoautotrophic and photoautotrophic bacteria [19-21]. In situ gene expression studies from environmental iron mat samples showed that genes predicted to be involved in glycogen synthesis in Zetaproteobacteria were upregulated within two minutes of $\mathrm{Fe}$ (II) addition [22]. A proteomics study of M. ferrooxydans PV-1 reported the presence of proteins involved in glycogen hydrolysis ([18], data set S1). These results are consistent with the hypothesis that glycogen can be both produced and utilized by Zetaproteobacteria.

Redox homeostasis under anoxic conditions can be maintained either by reduction of electron acceptors other than oxygen or by fermentation. Based on the presence of genes encoding for NirS and NirK type nitrite reductases in zetaproteobacterial metagenomes from iron mats at Loihi Seamount, Singer et al. [23] speculated that Zetaproteobacteria may be able to reduce nitrite. None of the genomes analyzed in our study were found to encode a NirS homolog; and only the Mariprofundus sp. EKF-M39 genome was found to encode a homolog of NirK, indicating the potential for nitrite reduction in this strain. Three of the analyzed Zetaproteobacteria genomes were found to contain a gene encoding a putative octaheme tetrathionate reductase (OTR, Table S2). In vitro studies on OTRs from $S$. oneidensis and Beggiatoa spp. showed its ability to reduce tetrathionate, nitrite, and hydroxylamine, though the physiological function of this protein remains unclear $[24,25]$. The presence of genes encoding putative OTRs therefore raises the possibility of nitrite, tetrathionate, and/or hydroxylamine reduction by Zetaproteobacteria. In addition, Mariprofundus sp. DIS-1 contains homologs of NapA and NapB, which suggests the possibility of nitrate reduction by this strain.

All the Zetaproteobacteria genomes analyzed in this study were found to contain both zinc-containing and iron-containing alcohol dehydrogenases, suggesting the possibility of fermentative production of alcohol to maintain redox homeostasis. The M. ferrooxydans EKF-M39 genome encodes a putative fermentative lactate dehydrogenase, which converts pyruvate to lactate while reoxidizing NADH to $\mathrm{NAD}^{+}$. Most of the Zetaproteobacteria genomes analyzed were found to encode for group 3b NAD-reducing $\mathrm{Ni}-\mathrm{Fe}$ hydrogenases [26]. These hydrogenases are known to be reversible, and can play a role in reoxidizing NADH/ NADPH. Three of the genomes also encode for group 4 $\mathrm{Ni}-\mathrm{Fe}$ hydrogen evolving hydrogenases [26], which can act as components of a formate hydrogen lyase complex linking formate oxidation by formate dehydrogenase (FdhH) to hydrogen production. Genes predicted to encode components of FdhH were not found in any of the Zetaproteobacteria genomes analyzed.

\section{Model for auxiliary anaerobic metabolism in M. ferrooxydans PV-1}

M. ferrooxydans PV-1 is the most studied Zetaproteobacteria isolate, and therefore we chose it as a model to characterize auxiliary anaerobic metabolism in Zetaproteobacteria. We tested the functionality of homologs for PflB, PflA, Pta, and AckA from M. ferrooxydans PV-1 (Table 1) using heterologous expression studies. Deletion mutants of S. oneidensis were chosen as the hosts for heterologous expression because $S$. oneidensis requires $\mathrm{Pfl}, \mathrm{Pta}$, and AckA for growth under anaerobic conditions [14, 27]. Heterologous expression of $p f l A$ and $p f l B$ from M. ferrooxydans PV-1 was able to complement growth of a $S$. oneidensis mutant lacking $p f B$ (Fig. 1a). Expression of both genes was required for growth, suggesting PflB from M. ferrooxydans was unable to interact with PflA from S. oneidensis (data not shown). Heterologous expression of pta and ackA from M. ferrooxydans PV-1 was also able to complement the anaerobic growth of a $S$. oneidensis mutant strain lacking pta and ackA (Fig. 1b). Consistent with previous observations, we were unable to detect evidence for heterotrophic growth of $M$. ferrooxydans PV-1 or utilization of other electron acceptors (Table S2), based on direct cell counts as described in Supplementary information.

An overview of auxiliary anaerobic metabolism in $M$. ferrooxydans PV-1 is presented in Fig. 1c. We propose that this metabolism is driven by endogenous glycogen produced during optimal aerobic growth conditions. M. ferrooxydans PV-1 is unlikely to utilize extracellular glycogen for either growth or survival. Utilization of extracellular glycogen requires hydrolysis of glycogen to glycans or glucose, which are transported inside the cell by specific transporter proteins for further metabolism. The M. ferrooxydans PV-1 genome does not encode for any known transporter proteins for either glycans or glucose. When oxygen is limiting, we propose that intracellular glycogen is 

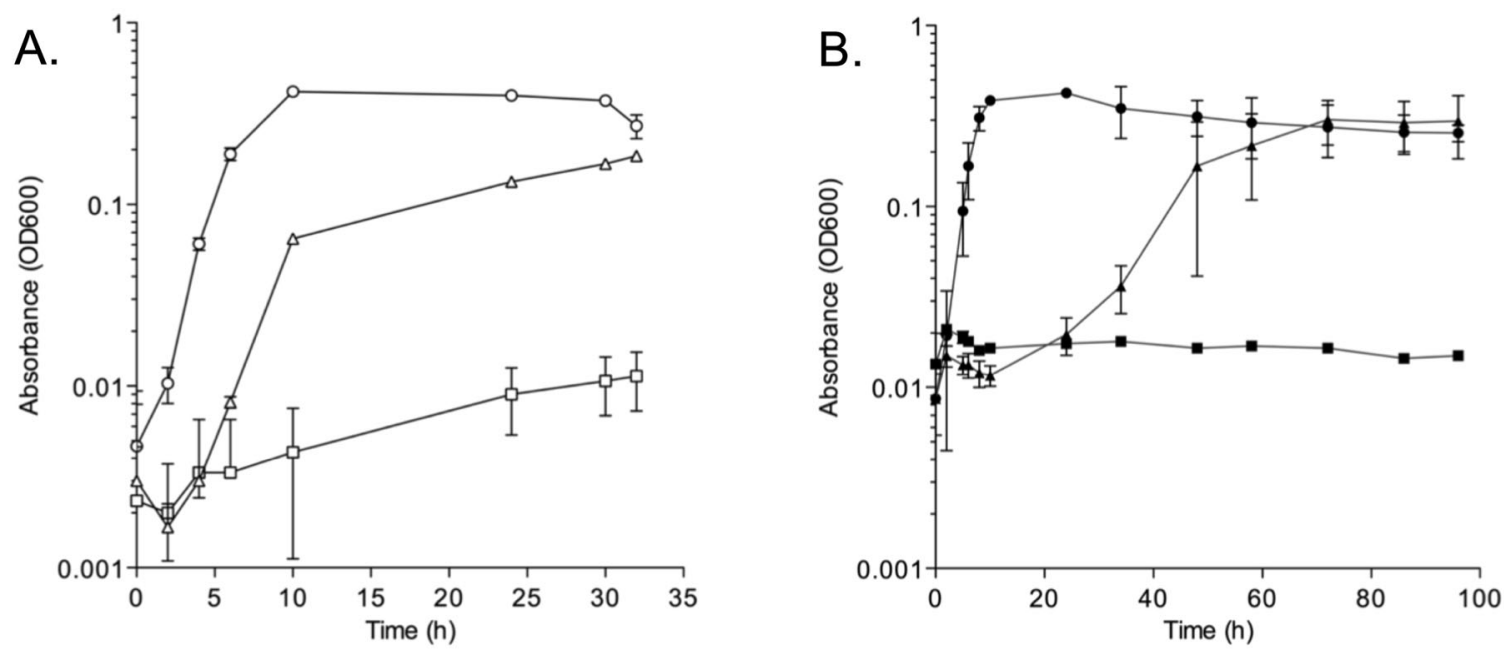

C.
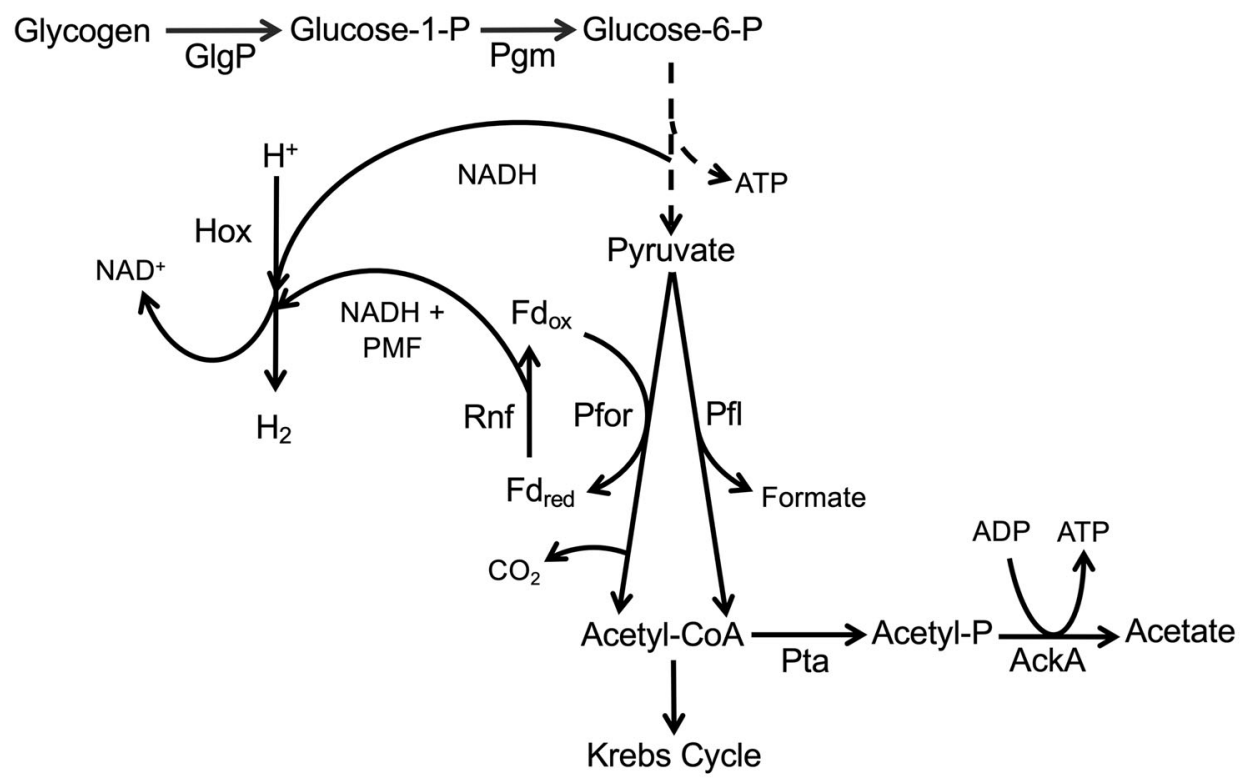

Fig. 1 Testing functionality of genes for homologs of pyruvate formate lyase, PflB; pyruvate formate lyase activating protein, PflA; phosphotransacetylase, Pta; and acetate kinase, AckA, from Mariprofundus ferrooxydans PV-1 by expressing them in corresponding deletion mutants of Shewanella oneidensis and overview of auxiliary anaerobic metabolism. a Growth of wild-type $S$. oneidensis MR-1 (O), $\triangle p f l B S$. oneidensis with empty pBBR1MCS2 ( $\square$ ), $\triangle p f l B S$. oneidensis with pBBR1MCS2::pflAB $B_{P V-1}(\Delta)$. b Growth of wild-type $S$. oneidensis MR-1 (O), $\Delta p t a \Delta a c k A$ S. oneidensis with

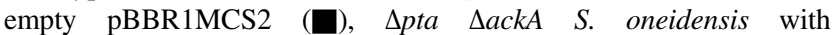
pBBR1MCS2::pta, $a c k A_{P V-I}(\boldsymbol{\Delta})$. All strains were grown on $20 \mathrm{mM}$ lactate as electron donor and $40 \mathrm{mM}$ fumarate as electron acceptor

hydrolyzed to glucose-1-phosphate, which is isomerized to glucose-6-phosphate for glycolysis (Fig. 1c). Further metabolism of glucose-6-phosphate during glycolysis produces NADH and ATP. Pyruvate produced from glycolysis is decarboxylated to acetyl CoA either by Pfl to produce formate, or by Pfor to produce reduced ferredoxin. Acetyl CoA is converted to acetyl phosphate by Pta, which is

under anoxic conditions. Error bars represent standard deviation of the mean from two independent experiments performed in triplicate. c Proposed auxiliary anaerobic metabolism in M. ferrooxydans PV-1 relying on endogenous glycogen for carbon source and reducing power. ATP is produced by substrate level phosphorylation during glycolysis and using AckA. Hox utilizes NADH produced during glycolysis along with NADH and proton motive force produced by Rnf to produce hydrogen to maintain redox homeostasis. Glycogen phosphorylase, GlgP; phosphoglucomutase, Pgm; pyruvate formate lyase, Pfl; pyruvate ferredoxin oxidoreductase, Pfor; Rnf complex consisting of six proteins RnfABCDGE, Rnf; NAD-reducing hydrogenase, Hox; phosphotransacetylase, Pta; acetate kinase, AckA.

subsequently converted to acetate and ATP by AckA. ATP can provide maintenance energy for survival and transport of metabolites. ATP hydrolysis can also be mediated by ATP synthase to generate PMF as previously demonstrated in Pseudomonas aeruginosa [28] and used to fuel flagellar motility, allowing cells to navigate toward oxic microenvironments. The Rnf complex oxidizes reduced 
ferredoxin to generate PMF and reduce NAD to NADH. NADH could then be reoxidized by NAD-reducing hydrogenases, while using PMF, to produce hydrogen to maintain the redox homeostasis under anoxic conditions.

Auxiliary anaerobic metabolism may differ in other neutrophilic $\mathrm{FeOB}$. Four genomes of $\mathrm{Fe}(\mathrm{II})$ oxidizing Betaproteobacteria (Sideroxydans lithotrophicus ES-1 [29], Gallionella capsiferriformans ES-2 [29], Ferriphaselus sp. R-1 [30], and Ferriphaselus amnicola OYT1 [31]) were found to have some, but not all of the components described in Fig. 1c and none found to encode a Pta homolog. From this preliminary analysis, $\mathrm{Fe}(\mathrm{II})$ oxidizing Betaproteobacteria appear to have a more limited capacity for auxiliary anaerobic metabolism, though additional genomes and more work with cultured isolates are warranted.

Although growth of Zetaproteobacteria requires Fe(II) oxidation coupled to aerobic respiration, our analysis is consistent with a role for auxiliary anaerobic metabolism in these bacteria. The presence of alternative PMF and ATP generating mechanisms could support survival strategies when $\mathrm{Fe}$ (II) oxidation is unavailable for Zetaproteobacteria. The mechanisms proposed can help direct future research to understand the ecology of Zetaproteobacteria and suggest that anaerobic metabolism can benefit even strict aerobes living near the oxic-anoxic interfaces.

Acknowledgements We thank Clara Chan and Sean McAllister for helpful discussions and feedback on the manuscript. This research was supported by the National Science Foundation Center for Dark Energy Biosphere Investigations (C-DEBI, Award OCE-0939564). This is CDEBI contribution \#519.

\section{Compliance with ethical standards}

Conflict of interest The authors declare that they have no conflict of interest.

Publisher's note Springer Nature remains neutral with regard to jurisdictional claims in published maps and institutional affiliations.

\section{References}

1. Emerson D, Rentz JA, Lilburn TG, Davis RE, Aldrich H, Chan C, et al. A novel lineage of proteobacteria involved in formation of marine Fe-oxidizing microbial mat communities. PloS ONE. 2007;2:667.

2. Davis RE, Stakes DS, Wheat CG, Moyer CL. Bacterial variability within an iron-silica-manganese-rich hydrothermal mound located off-axis at the cleft segment, Juan de Fuca Ridge. Geomicrobiol J. 2009;26:570-80.

3. Edwards KJ, Glazer BT, Rouxel OJ, Bach W, Emerson D, Davis $\mathrm{RE}$, et al. Ultra-diffuse hydrothermal venting supports $\mathrm{Fe}$ oxidizing bacteria and massive umber deposition at $5000 \mathrm{~m}$ off Hawaii. ISME J. 2011;5:1748-58.

4. McAllister SM, Davis RE, McBeth JM, Tebo BM, Emerson D, Moyer CL. Biodiversity and emerging biogeography of the neutrophilic iron-oxidizing Zetaproteobacteria. Appl Environ Microbiol. 2011;77:5445-57.
5. Fleming EJ, Davis RE, McAllister SM, Chan CS, Moyer CL, Tebo BM, et al. Hidden in plain sight: discovery of sheathforming, iron-oxidizing Zetaproteobacteria at Loihi Seamount, Hawaii, USA. FEMS Micro Ecol. 2013;85:116-27.

6. Borch T, Kretzschmar R, Kappler A, Cappellen PV, Ginder-Vogel $\mathrm{M}$, Voegelin A, et al. Biogeochemical redox processes and their impact on contaminant dynamics. Environ Sci Technol. 2009; 44:15-23.

7. McBeth JM, Little BJ, Ray RI, Farrar KM, Emerson D. Neutrophilic iron-oxidizing "Zetaproteobacteria" and mild steel corrosion in near shore marine environments. Appl Environ Microbiol. 2011;77:1405-12.

8. Field EK, Sczyrba A, Lyman AE, Harris CC, Woyke T, Stepanauskas R, et al. Genomic insights into the uncultivated marine Zetaproteobacteria at Loihi Seamount. ISME J. 2015;9: 857-70.

9. Makita H, Tanaka E, Mitsunobu S, Miyazaki M, Nunoura T, Uematsu K, et al. Mariprofundus micogutta sp. nov., a novel ironoxidizing zetaproteobacterium isolated from a deep-sea hydrothermal field at the Bayonnaise knoll of the Izu-Ogasawara arc, and a description of Mariprofundales ord. nov. and Zetaproteobacteria classis nov. Arch Microbiol. 2016;20:1-12.

10. McAllister SM, Moore RM, Gartman A, Luther GW, Emerson D, Chan CS. The Fe(II)-oxidizing Zetaproteobacteria: historical, ecological and genomic perspectives. FEMS Microbiol Ecol. 2019;95:fiz015.

11. Wagner AF, Frey M, Neugebauer FA, Schäfer W, Knappe J. The free radical in pyruvate formate-lyase is located on glycine-734. Proc Natl Acad Sci. 1992;89:996-1000.

12. Hasona A, Kim Y, Healy FG, Ingram LO, Shanmugam KT. Pyruvate formate lyase and acetate kinase are essential for anaerobic growth of Escherichia coli on xylose. J Bacteriol. 2004; 186:7593-600.

13. Doi Y, Ikegami Y. Pyruvate formate-lyase is essential for fumarate-independent anaerobic glycerol utilization in the Enterococcus faecalis strain W11. J Bacteriol. 2014;196:2472-80.

14. Flynn CM, Hunt KA, Gralnick JA, Srienc F. Construction and elementary mode analysis of a metabolic model for Shewanella oneidensis MR-1. Biosysystems. 2012;107:120-8.

15. Wolfe AJ. The acetate switch. Microbiol Mol Biol Rev. 2005;69: $12-50$.

16. Biegel E, Müller V. Bacterial Na+-translocating ferredoxin: NAD + oxidoreductase. Proc Natl Acad Sci. 2010;107:18138-42.

17. Schmehl M, Jahn A, zu Vilsendorf AM, Hennecke S, Masepohl B, Schuppler $\mathrm{M}$, et al. Identification of a new class of nitrogen fixation genes in Rhodobacter capsalatus: a putative membrane complex involved in electron transport to nitrogenase. Mol Gen. 1993;241:602-15.

18. Barco RA, Emerson D, Sylvan JB, Orcutt BN, Meyers MEJ, Ramírez GA, et al. New insight into microbial iron oxidation as revealed by the proteomic profile of an obligate ironoxidizing chemolithoautotroph. Appl Environ Microbiol. 2015; 81:5927-37.

19. Stal LJ, Moezelaar R. Fermentation in cyanobacteria. FEMS Microbiol Rev. 1997;21:179-211.

20. Oren A, Shilo M. Anaerobic heterotrophic dark metabolism in the cyanobacterium Oscillatoria limnetica: sulfur respiration and lactate fermentation. Arch Microbiol. 1979;122:77-84.

21. Beudeker RF, De Boer W, Kuenen JG. Heterolactic fermentation of intracellular polyglucose by the obligate chemolithotroph Thiobacillus neapolitanus under anaerobic conditions. FEMS Microbiol Lett. 1981;12:337-42.

22. McAllister SM, Polson SW, Butterfield DA, Glazer BT, Sylvan JB, Chan CS. Validating the Cyc2 neutrophilic Fe oxidation pathway using meta-omics of Zetaproteobacteria iron mats at marine hydrothermal vents. 2019. https://doi.org/10.1101/722066. 
23. Singer E, Heidelberg JF, Dhillon A, Edwards KJ. Metagenomic insights into the dominant Fe (II) oxidizing Zetaproteobacteria from an iron mat at Lōihi, Hawaii. Front Microbiol. 2013;4:52.

24. Atkinson SJ, Mowat CG, Reid GA, Chapman SK. An octaheme $c$ type cytochrome from Shewanella oneidensis can reduce nitrite and hydroxylamine. FEBS Lett. 2007;581:3805-8.

25. MacGregor BJ, Biddle JF, Siebert JR, Staunton E, Hegg EL, Matthysse AG, et al. Why orange Guaymas Basin Beggiatoa spp. are orange: single-filament-genome-enabled identification of an abundant octaheme cytochrome with hydroxylamine oxidase, hydrazine oxidase, and nitrite reductase activities. Appl Environ Microbiol. 2013;79:1183-90.

26. Vignais PM, Billoud B, Meyer J. Classification and phylogeny of hydrogenases. FEMS Microbiol Rev. 2001;25:455-501.

27. Hunt KA, Flynn JM, Naranjo B, Shikhare ID, Gralnick JA. Substrate-level phosphorylation is the primary source of energy conservation during anaerobic respiration of Shewanella oneidensis strain MR-1. J Bacteriol. 2010;192:3345-51.

28. Glasser NR, Kern SE, Newman DK. Phenazine redox cycling enhances anaerobic survival in Pseudomonas aeruginosa by facilitating generation of ATP and a proton-motive force. Mol Microbiol. 2014;92:399-412.

29. Emerson D, Moyer C. Isolation and characterization of novel ironoxidizing bacteria that grow at circumneutral $\mathrm{pH}$. Appl Environ Microbiol. 1997;63:4784-92.

30. Krepski ST, Hanson TE, Chan CS. Isolation and characterization of a novel biomineral stalk-forming iron-oxidizing bacterium from a circumneutral groundwater seep. Environ Microbiol. 2012;14:1671-80.

31. Kato S, Krepski S, Chan C, Itoh T, Ohkuma M. Ferriphaselus amnicola gen. nov., sp. nov., a neutrophilic, stalk-forming, ironoxidizing bacterium isolated from an iron-rich groundwater seep. Int J Syst Evol Microbiol. 2014;64:921-5. 